\title{
On-board hybrid magnetometer of NASA CHARM-II rocket: principle, design and performances
}

\author{
C. Coillot $^{1}$, J. Moutoussamy ${ }^{1}$, G. Chanteur ${ }^{1}$, P. Robert ${ }^{1}$, and F. Alves ${ }^{2}$ \\ ${ }^{1}$ LPP/CNRS/UPMC/Ecole Polytechnique, Route de Saclay, 91128 Palaiseau, France \\ ${ }^{2}$ LGEP/CNRS/Paris XI, 11 rue Joliot Curie, 91192 GIF sur Yvette, France \\ Correspondence to: C. Coillot (christophe.coillot@lpp.polytechnique.fr)
}

Received: 15 November 2012 - Revised: 24 June 2013 - Accepted: 27 June 2013 - Published: 6 August 2013

\begin{abstract}
We present a hybrid tri-axes magnetometer designed to measure weak magnetic fields in space from DC (direct current) up to a few $\mathrm{kHz}$ with a better sensitivity than fluxgate magnetometers at frequencies above a few $\mathrm{Hz}$. This magnetometer combines a wire-wound ferromagnetic ribbon and a classical induction sensor. The nature of the wire-wound ferromagnetic ribbon sensor, giant magneto-impedance or magneto-inductance, is discussed. New configurations of wire-wound ferromagnetic ribbon sensors based on closed magnetic circuits are suggested and the hybrid sensor is described. The electronic conditioning of the wire-wound ribbon makes use of an alternating bias field to cancel the offset and linearize the output. Finally we summarize the main performances of the hybrid magnetometer and we discuss its advantages and drawbacks. A prototype has been built and was part of the scientific payload of the NASA rocket experiment CHARM-II (Correlation of High Frequency and Auroral Roar Measurements) launched in the auroral ionosphere. Unfortunately the launch campaign ended without any noticeable magnetic event and the rocket was eventually launched on 16 February 2010, through a very quiescent arc in the magnetic cusp and no wave activity was detected at frequencies observable by the hybrid magnetometer.
\end{abstract}

\section{Introduction}

The hybrid magnetometer aims to cover a frequency range of magnetic field measurement from DC up to a few $\mathrm{kHz}$ for plasma waves study in space and especially the investigation of the Langmuir and upper hybrid waves in the auroral ionosphere (Samara et al., 2004) in the context of the NASA rocket CHARM-II (Correlation of High Frequency and Auroral Roar Measurements). This magnetometer extends the magnetic field measurement of the induction sensor to the low frequencies $(<1 \mathrm{~Hz})$ in order to ensure a redundancy with fluxgate magnetometers which are onboard rockets and spacecraft for plasma wave studies. The ultimate objective is to reach a noise equivalent magnetic induction comparable to the one of the fluxgate used in space experiments (about $5 \mathrm{pT} \mathrm{Hz} / \sqrt{\mathrm{Hz}}$ at $1 \mathrm{~Hz}$ for Oersted magnetometer from Hika et al., 1996). This novel hybrid sensor combines induction sensor and a magneto-impedance sensor and covers magnetic field measurement from DC up to $20 \mathrm{kHz}$. In this paper we will focus on the DC part of the hybrid sensor which is ob- tained using a kind of giant magneto-impedance (GMI). The GMI effect is the large variation of the AC impedance of a magnetic conductor with an applied DC or ultra-low frequency magnetic field. It has been evidenced in ferromagnetic wires by Harrison et al. (1936), in microwires by Panina and Mohri (1994) and Panina et al. (1994), in ribbons and sandwiches by Hika et al. (1996), Panina et al. (1994) and Morikawa et al. (1997), and more recently in transverse or longitudinal wire-wound ferromagnetic ribbons by Moutoussamy et al. (2009). Sensors making use of the GMI effect are characterized by their robustness, small mass and high sensitivity to weak magnetic fields which make them suitable for many applications. The ambient magnetic field to be measured modifies the dynamic magnetization of the magnetic material through its magnetic susceptibility tensor. At high frequency the modification of susceptibility due to the magnetic field is accompanied by a skin depth change resulting in a strong impedance variation. 


\section{Principle of the wire-wound ferromagnetic ribbon magnetic sensor}

\subsection{Discussion about the nature of the wire-wound ferromagnetic ribbon sensor: magneto-inductance or} giant magneto-impedance?

The skin effect is usually invoked to explain the GMI effect but, in fact, the skin effect in sandwich GMI is more complicated than usually thought due to the lateral skin effect which occurs first in the copper conductor as described by Belevitch (1971). Simulation results in the middle section of a ferro/copper/ferro sandwich, presented in Fig. 1, illustrates the lateral skin effect at $1 \mathrm{MHz}$ on the cross section of an infinite copper ribbon (width $=200 \mu \mathrm{m}$, thickness $=20 \mu \mathrm{m}$ ); the current density is expelled from the centre of the conductor to the lateral edges of the ribbon. However the skin effect in a ferromagnetic ribbon occurs as expected (the current density is confined into the skin thickness) when the relative permeability is sufficiently large, due to the edge effect discussed by Garcia-Arribas et al. (2008). Nevertheless the real GMI sandwich exhibits a poor current conduction between the copper ribbon and the magnetic material ribbons. The current flowing through the ferromagnetic ribbon is negligible, this experimental fact has led Moutoussamy et al. (2009) to implement an excitation by coils. However it should be noticed that the magnetic field radiated by the current flowing through the copper ribbon generates eddy currents inside the ferromagnetic ribbon, these are neglected in this article.

Let us now examine the effect of an external magnetic field on the magnetization of the ferromagnetic ribbon. When a magnetic field $\boldsymbol{H}$ is applied to a ferromagnetic ribbon, as shown in Fig. 2, the magnetization $\boldsymbol{M}$, which makes an angle $\theta$ with the $x$ axis, is modified in two ways, by its rotation and through the displacement of the domain walls. When considering only the rotation of the magnetization the susceptibility tensor is obtained by solving the Landau-Lifschitz-Gilbert (LLG) equation governing the evolution of the magnetization $\boldsymbol{M}$ :

$\frac{d \boldsymbol{M}}{\mathrm{d} t}=-\gamma \mu_{0} \boldsymbol{M} \times \boldsymbol{H}_{\text {eff }}+\alpha \frac{\boldsymbol{M}}{M_{\mathrm{s}}} \times \frac{\mathrm{d} \boldsymbol{M}}{\mathrm{d} t}$,

where $\gamma$ corresponds to the gyromagnetic ratio, $\alpha$ measures the damping, and $M_{\mathrm{S}}$ is the saturated magnetization. The use of the LLG equation is based on the hypothesis of a single magnetic domain structure which is a rough assumption as the magnetic structure of the ribbon is obviously multidomain.

The effective magnetic field $\boldsymbol{H}_{\text {eff }}$ is derived, accordingly to Eq. (2), by minimizing the free energy, defined by Eq. (3), which is the sum of the magneto-crystalline anisotropic energy, the Zeeman energy, and the demagnetizing energy.

$$
\boldsymbol{H}_{\text {eff }}=-\frac{1}{\gamma \mu_{0} M_{\mathrm{S}}} \frac{\partial W}{\partial \theta}
$$

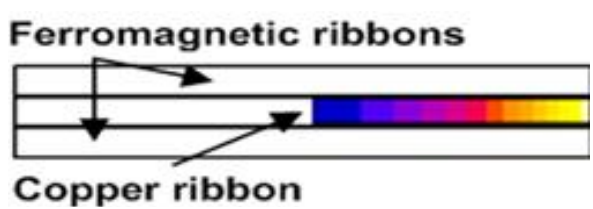

Figure 1. Current density distribution inside the middle half cross section of a ferro/copper/ferro sandwich at $1 \mathrm{MHz}$ (blue to yellow corresponds to a ratio on the current density magnitude from 1 to $4)$.

$W=K \sin ^{2} \theta-\mu_{0} \boldsymbol{H} \cdot \boldsymbol{M}-\frac{1}{2} \mu_{0} \boldsymbol{H}_{\mathbf{d}} \cdot \boldsymbol{M}$

$K$ is the anisotropy constant, $\boldsymbol{H}_{\mathrm{d}}$ the demagnetizing field, and $\mu_{0}$ the vacuum permeability. The resolution of these equations is beyond the scope of this article but is detailed for ferromagnetic ribbons in Moutoussamy (2009); it allows one to compute the susceptibility tensor $\chi$ linking the fluctuation $\boldsymbol{m}$ of the magnetization to the driving magnetic field $\boldsymbol{h}_{\mathrm{exc}}$ at pulsation $\omega_{\text {exc }}$.

$\boldsymbol{m}=\chi \boldsymbol{h}_{\mathrm{exc}}$

In the case of a ferromagnetic ribbon, the susceptibility tensor will have the following components in the $x-y$ plane defined in Fig. 2:

$\chi=\left|\begin{array}{cc}\chi_{x x}\left(H, \omega_{\text {exc }}\right) & \chi_{x y}\left(H, \omega_{\text {exc }}\right) \\ \chi_{y x}\left(H, \omega_{\text {exc }}\right) & \chi_{y y}\left(H, \omega_{\text {exc }}\right)\end{array}\right|$.

The dependency of each component of the susceptibility tensor upon the ambient magnetic field $\boldsymbol{H}$ gives rise to a possible measurement of the magnetic field, as demonstrated by Moutoussamy et al. (2009), even if there is no skin effect when the frequency of the excitation current is as low as a few $\mathrm{kHz}$. The current excitation at high frequency used in classical GMI discussed by Panina and Mohri (1994) and Hika et al. (1996) allows the onset of the skin effect which enhances the impedance variation caused by the susceptibility variation. The ferromagnetic ribbon of the hybrid sensor is made of Ultraperm manufactured by VAC (2002), a high permeability soft ferromagnetic; it has a thickness of $40 \mu \mathrm{m}$, and is supplied at a frequency higher than $10 \mathrm{kHz}$. At this frequency the skin depth of the ribbon is much smaller than its middle thickness and we can consider that eddy currents inside the ferromagnetic ribbon will be confined into the skin depth that will be modified by the magnetic field.

Moreover, by considering the frequency regime distinction between magneto-inductance and GMI in ferromagnetic wire proposed by Panina et al. (1994), the wire-wound ferromagnetic ribbon could be categorized as a GMI sensor. Nevertheless the use of a winding appears as an alternative way to enhance the susceptibility variation caused by the magnetic field. The diagonal components of the susceptibility tensor 


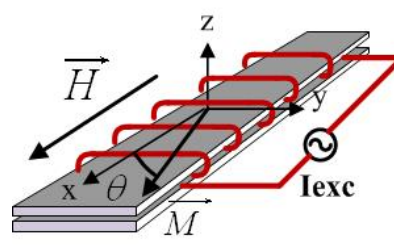

a)

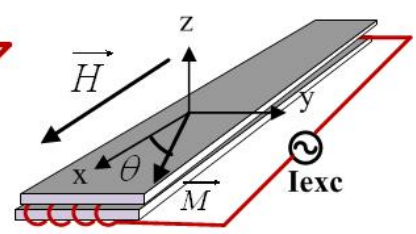

b)

Figure 2. Transverse (a) and longitudinal (b) wire-wound ferromagnetic ribbon constituting the magnetic sensor under the ambient magnetic field $(\boldsymbol{H})$.

are involved in the transverse and longitudinal wire-wound ferromagnetic ribbons constituting the magnetic sensor (cf. Fig. 2a, b) meanwhile its off-diagonal components are involved in GMIs using a pickup coil and named off-diagonal in Malatek and Kraus (2010). It should be noted that one or two ribbons can be used in both cases, transverse or longitudinal. The main advantage of using two ferromagnetic ribbons is to close the driving magnetic field lines thus allowing us to reduce the driving current, but in each case a magnetoimpedance effect will occur. Let us notice the availability of miniaturized commercial magneto-inductive sensors apparently similar to the wire-wound transverse GMI with a single ribbon, although Leuzinger and Taylor (2010) gave no detail on their design.

\subsection{Impedance behaviour of the transversal wire-wound $\mathrm{GMI}$}

The coil of the wire-wound GMI sandwich is driven by an excitation current $I_{\text {exc }}$ at frequency $f_{\text {exc }}$. When a magnetic field $\boldsymbol{H}$ is applied to a transverse wire-wound GMI, the modulus of the impedance $\left|Z\left(H, f_{\text {exc }}\right)\right|$ starts to increase smoothly before reaching a maximum and then starts to decrease asymptotically to zero as shown in Fig. 3a. The intrinsic sensitivity of the magnetic sensor defined by

$S_{\mathrm{I}}=\frac{\partial\left|Z\left(H, f_{\mathrm{exc}}\right)\right|}{\partial H}$

is plotted in Fig. 3b. The value of the applied magnetic field corresponding to the largest negative slope of $\left|Z\left(H, f_{\text {exc }}\right)\right|$, i.e. to the maximal intrinsic sensitivity, is close to the anisotropy magnetic field $H_{\text {ani }}$ resulting from a combination between the magneto-crystalline and shape anisotropies (Moutoussamy, 2009) of the ferromagnetic ribbon, where the shape anisotropy dominates because of the advantageous shape ratio of the ribbon.

\subsection{Elements of impedance modelling}

Let us consider a single ferromagnetic ribbon wound by a $N$ turns coil. When the coil is driven by a current $I_{\mathrm{exc}}$ at pulsation $\omega_{\text {exc }}$, the impedance modulus can be roughly expressed

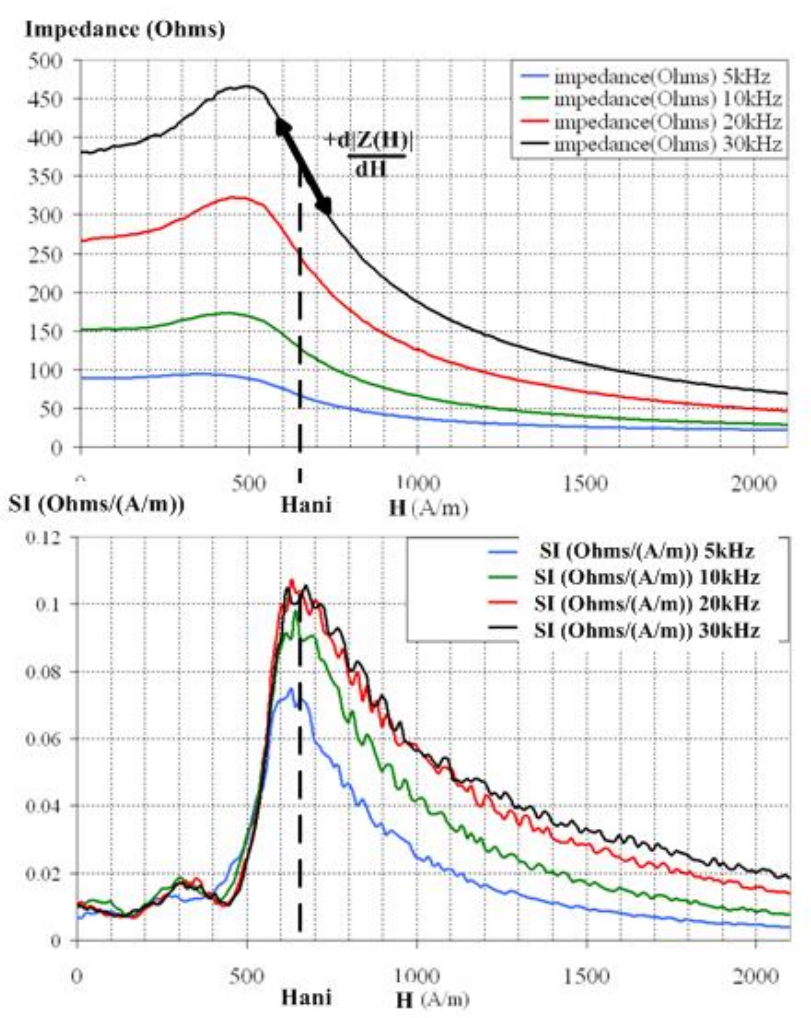

Figure 3. Variations with the magnetic field $H$ of the modulus of the impedance (upper frame) and intrinsic sensitivity (lower frame) of a longitudinal wire-wound magnetic ribbon.

by the following equation if the resistance of the coil can be neglected:

$\left|Z\left(H, \omega_{\mathrm{exc}}\right)\right|=\omega_{\mathrm{exc}} \frac{N^{2} S_{\text {ribbon }} \mu_{\mathrm{app}}\left(H, \omega_{\mathrm{exc}}\right)}{l_{\text {ribbon }}}$,

where $S_{\text {ribbon }}$ is the section of the ferromagnetic ribbon, $l_{\text {ribbon }}$ its length, and $\mu_{\mathrm{app}}\left(H, \omega_{\mathrm{exc}}\right)$ is the apparent permeability of the ribbon along the $x$ direction which can be derived from component $\chi_{x x}\left(H, \omega_{\text {exc }}\right)$ of the susceptibility tensor (Eq. 5) as the external magnetic field to be measured and the excitation magnetic field are both aligned with $x$. The susceptibility $\chi_{x x}$ is assumed to be complex in order to take into account losses in the ferromagnetic ribbon.

$\mu_{\text {app }}\left(H, \omega_{\text {exc }}\right)=\frac{\chi_{x x}\left(H, \omega_{\text {exc }}\right)-1}{1+N_{x} \chi_{x x}\left(H, \omega_{\text {exc }}\right)}$

The apparent permeability $\mu_{\text {app }}$ depends upon the susceptibility $\chi_{x x}$ but also on the shape of the ferromagnetic ribbon through its demagnetizing factor $N_{x}$ in the sensing direction. Exact calculations of magnetometric demagnetizing factors of ribbons are given by Aharoni (1998). 


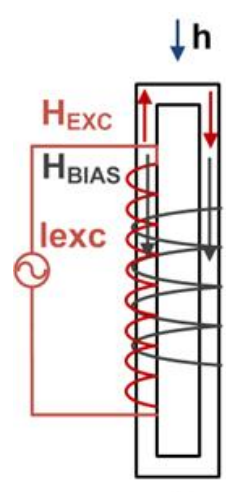

a)

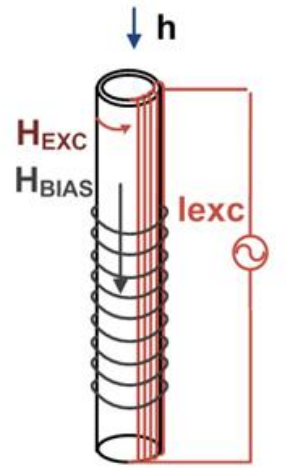

b)

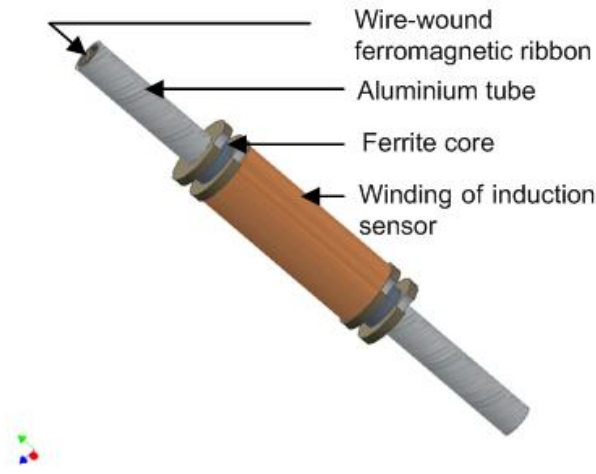

c)

Figure 4. Racetrack (a) and cylindrical (b) wire-wound ferromagnetic core. (c) Hybrid sensor: induction sensor and wire-wound GMI inside an aluminium tube.

\subsection{Intrinsic sensitivity}

The full expression of the impedance requires a cumbersome analysis to design the sensor, nevertheless Moutoussamy et al. (2007) have made a linear analysis by considering the magnetic field to be measured $(h)$ as a small fluctuation around a magnetic field bias $H=H_{\text {bias }}$. The optimal value of intrinsic sensitivity is obtained when $H_{\text {bias }}=H_{\text {ani }}$. Thus the impedance modulus, which is a function of both the bias magnetic field and the magnetic field to be measured $(h)$, can be expressed by the equation below.

$\left|Z\left((H+h), f_{\text {exc }}\right)\right|=\left|Z\left(H_{\text {bias }}, f_{\text {exc }}\right)\right|+\left.\frac{\partial\left|Z\left(H, f_{\text {exc }}\right)\right|}{\partial H}\right|_{H=H_{\text {bias }}}$

$$
\times h \sin (\omega t)
$$

The optimal intrinsic sensitivity, (at $H=H_{\text {bias }}$ ), defined by

$S_{\mathrm{I}}=\left.\frac{\partial\left|Z\left(H, f_{\text {exc }}\right)\right|}{\partial H}\right|_{H=H_{\text {bias }}}$

has been used by Moutoussamy et al. (2007) and Dumay et al. (2012) to characterize the ability of the GMI sensor to measure a weak magnetic field, it can be expressed as follows by combining Eqs. (7) and (10):

$S_{\text {I }}=\left.\omega_{\text {exc }} \frac{N^{2} S_{\text {ribbon }}}{l_{\text {ribbon }}} \frac{\partial \mu_{\text {app }}\left(H, f_{\text {exc }}\right)}{\partial H}\right|_{H=H_{\text {bias }}}$.

It results from the partial derivative of the apparent permeability with respect to $H$ that the lower the demagnetizing factor is, the higher the sensitivity that will be achieved. This basic fact explains why most of the efficient GMI sensors reported in the literature exhibit advantageous demagnetizing factors due to a large length/diameter ratio. The design of a GMI sensor, looking for the best material and shape of the ferromagnetic ribbon, and the optimal parameters $(N$, $\left.I_{\text {exc }}, \omega_{\text {exc }}, H_{\text {bias }}\right)$ to get the maximal intrinsic sensitivity, is an experimental task as emphasized by Moutoussamy et al. (2009).

\section{Wire-wound GMI ribbon for hybrid sensor design}

New configurations of wire-wound GMI sensors based on closed magnetic circuits, presented in Fig. 4a and b, reduce the leakage of the magnetic flux created by the excitation current. This aspect is of great importance in order to combine the GMI sensor with a search coil. The GMI in Fig. 4a reminds the classical racetrack fluxgate reminded by Ripka (2003), while the GMI in Fig. 4b, which consists of a wirewound sheet of ferromagnetic ribbon rolled around to make a cylindrical muff, reminds the orthogonal fluxgate studied by Paperno et al. (2008) even if the wire-wound GMI does not use a pickup coil since the measurement is done directly on the driving coil. Moreover the ferromagnetic material is not driven at saturation. Even if these "closed magnetic path configurations" exhibit interesting properties, the transverse configuration of GMI illustrated in Fig. 2a has been preferred for the rocket instrument due to its simplicity of manufacturing. The transverse wire-wound GMI sensor used for the experiment had 100 turns both for excitation and bias coils, the ribbon made of Fe-Ni alloy (Ultraperm manufactured by VAC, 2002) had a length of $120 \mathrm{~mm}$, a width equal to $4 \mathrm{~mm}$, and a thickness of $40 \mu \mathrm{m}$. The excitation coil was wound around one of the two ribbons, while the bias coil surrounded the two ribbons.

In the transverse wire-wound configuration with a closed magnetic path, the excitation and bias driving current will create an AC magnetic field. This magnetic field will have leakages flux which are reduced by the addition of an aluminium tube of $1 \mathrm{~mm}$ thickness since the eddy current generated by the AC magnetic field expels the magnetic field outside the tube (this effect is stronger at frequencies for which the skin depth is comparable to the thickness of the tube). Finally, the GMI sensor surrounded by an aluminium tube is inserted inside a hollow ferrite magnetic core on which the induction sensor (also known as search coil) is built as shown in Fig. 4c. Thus, the AC magnetic field expelled from 
the aluminium tube $(120 \mathrm{~mm}$ length, $6 \mathrm{~mm}$ internal diameter and $7.95 \mathrm{~mm}$ external diameter) is caught by the surrounding ferrite core of the induction sensor (Tumanski, 2007 and Coillot and Leroy, 2012). The ferromagnetic core of the induction sensor is a hollow tube made of a high permeability Mn-Zn ferrite $\left(\mu_{\mathrm{r}}>2500\right)$ having a length of $50 \mathrm{~mm}$, an internal diameter of $8 \mathrm{~mm}$, and an external diameter of $10 \mathrm{~mm}$. The winding consists of 9600 turns of a copper wire having a diameter equal to $70 \mu \mathrm{m}$ in order to fulfill the detection requirement of in situ electromagnetic waves specified by a noise equivalent magnetic induction (NEMI) lower than $100 \mathrm{fT} / \sqrt{\mathrm{Hz}}$ at few $\mathrm{kHz}$. The induction sensor was combined with a low-noise feedback flux preamplifier (voltage and current input noise were respectively $6 \mathrm{nV} / \sqrt{\mathrm{Hz}}$ and $100 \mathrm{fA} / \sqrt{\mathrm{Hz}}$ ) to achieve the required NEMI. The lownoise amplifier implemented for the experiment was similar to the one described by Seran and Fergeau (2005), making use of a dual JFET transistor type LS-U404 from Linear Systems. A usual flux feedback, see for example Seran and Fergeau (2005) or Coillot and Leroy (2012), uses the output of the low-noise amplifier to generate a flux opposite to the measured one in order to flatten the transfer function. It has been checked experimentally that the internal aluminium tube does not increase the NEMI of the induction sensor above the specified level of $100 \mathrm{fT} / \sqrt{\mathrm{Hz}}$.

\section{Signal conditioning principle}

Let us consider a bias field $H_{\text {bias }}$ applied to the ferromagnetic ribbon. Accordingly to Eq. (9) the output voltage of the wirewound transverse GMI supplied with a current $I_{\mathrm{exc}}$ is written

$V_{\mathrm{GMI}}=-\left[\left|Z\left(H_{\text {bias }}\right)\right|+S_{\mathrm{I}}\left(H_{\text {bias }}\right) h \sin (\omega t)\right] I_{\mathrm{exc}} \sin \left(\omega_{\mathrm{exc}} t\right)$

The implemented signal conditioning takes advantage of the symmetry of the modulus of the impedance, $|Z(-H)|=$ $|Z(H)|$. For two opposite bias fields $\pm H_{\text {bias }}$ the offsets $Z\left( \pm H_{\text {bias }}\right) I_{\text {exc }} \cos \left(\omega_{\text {exc }} t\right)$ are equal meanwhile the slopes $S_{\mathrm{I}}\left( \pm H_{\text {bias }}\right)$ are opposite. If the bias field is modulated at a bias frequency $f_{\text {bias }}$ then the magnetic field $h \sin (\omega t)$ to be measured is modulated twice: at the excitation frequency and at the bias frequency (plus bias harmonics) while the impedance will not be modulated by the bias field. We chose $f_{\text {exc }}=100 \mathrm{kHz}$ and $f_{\text {bias }}=100 \mathrm{~Hz}$. If we assume a sinusoidal waveform of the excitation $I_{\mathrm{exc}}$ at $f_{\mathrm{exc}}$ and a square waveform of the bias field, the voltage of the GMI can be written

$V_{\mathrm{GMI}}=\left(I_{\mathrm{exc}} \cos \left(\omega_{\mathrm{exc}} t\right)\right)$

$\times\left(\left(Z\left(H_{\text {bias }}\right)+\sum_{k}^{\infty} S_{\mathrm{I}}(H) \times h \sin (\omega t) \frac{4}{k \pi} \cos \left(k \omega_{\text {bias }} t\right)\right)\right.$.

Consequently, the magnetic field signal $(h)$ can be retrieved by using a double demodulation at both $f_{\text {exc }}$ and $f_{\text {bias }}$ while the offset value (corresponding at $Z(H)$ ) will be easily suppressed. It should be noticed that the phase of demodulation at $f_{\text {exc }}$ should be adjusted experimentally to get the maximum sensitivity. For wire-wound GMI supplied at low frequency, through the voltage source in series with the resistance $R s$ (cf. Fig. 5), the demodulation in phase with the inductive part is optimum. A schematic explanation of the electronic principle that is used to cancel the offset is presented in Fig. 5.

The demodulation is realized using multipliers based on a switch matrix (cf. Fig. 5). A switch matrix, based on DG419 switches, is used to multiply the signal by \pm 1 with low added noise thanks to their extremely low $R_{\mathrm{DSON}}$. This way of demodulating is more efficient in terms of power consumption than demodulation using analog multipliers (for lownoise multipliers, like AD835, current consumption is about $16 \mathrm{~mA}$ ). One can notice that demodulation using peak detector, used by Dumay et al. (2012), offers also an efficient way to demodulate the GMI signal even if it is not applicable to the GMI with alternating bias. We will now expose the principle of this electronic conditioning. For this purpose, we will consider only the fundamental of the bias magnetic field. If we examine the GMI voltage after the primary amplification (A1) in the frequency domain (Fig. 6), it appears that the signal $(h)$ is twice modulated around $f_{\text {exc }} \pm f_{\text {bias }}$ while the offset remains at $f_{\text {exc }}$.

Then, after a first demodulation at $f_{\text {exc }}$ (with an optimal phase difference with excitation current determined experimentally) the offset signal is moved at null frequency while the signal $(h)$ is moved around $f_{\text {bias }}$ (cf. Fig. 7).

Next the offset is removed using simple high-pass filtering (Fig. 8) and finally the signal is moved in its original band (Fig. 9) thanks to the demodulation at $f_{\text {bias }}$.

Surprisingly, this offset cancellation technique seems very similar to the one used by Sasada (2002) for fluxgate in orthogonal mode.

\section{Hybrid magnetometer performances}

The characterization in terms of intrinsic sensitivity has been achieved for the GMI part of each hybrid sensor (Fig. 10a) in order to find the best compromise between a high sensitivity $S_{\mathrm{I}}$, a high dynamic range and a low current consumption. In order to determine the minimum dynamic range in the Earth's magnetic field, characterizations of $S_{\mathrm{I}}$ have been done in 3 directions (Fig. 10a): parallel to Earth's magnetic field, anti-parallel and orthogonal. This explains the shift of the curves around the centered $S_{\text {I }}$ curve which corresponds to orthogonal position. The electronic conditioning, which includes an offset cancellation technique offers an intrinsic linearization, demonstrated in Fig. 10b. The linearity range, approximatively $\pm 25 \mu \mathrm{T}$ would be significantly improved by using a flux feedback. The transfer function (Fig. 10c) obtained for the GMI part of the hybrid sensor (picture label in 


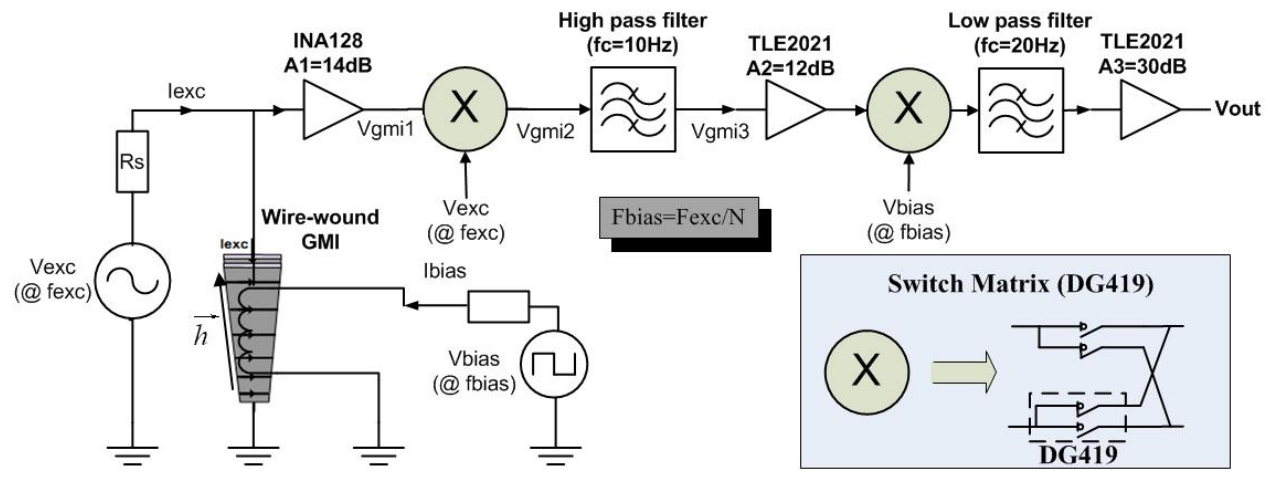

Figure 5. Signal conditioning principle.

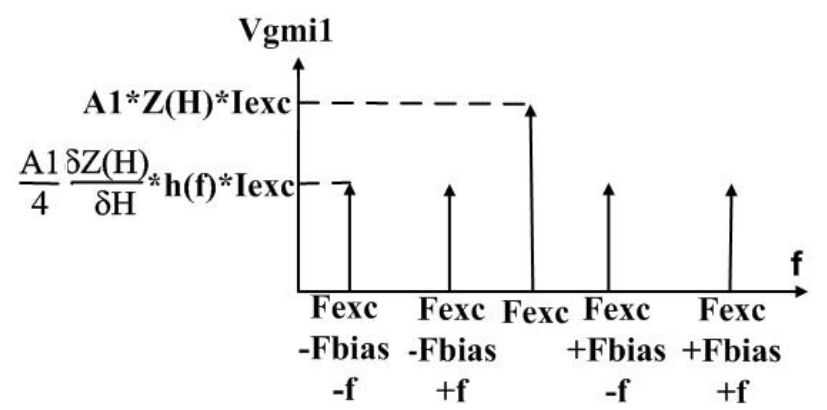

Figure 6. Frequency domain of GMI signal (Vgmi1) after amplification A1.

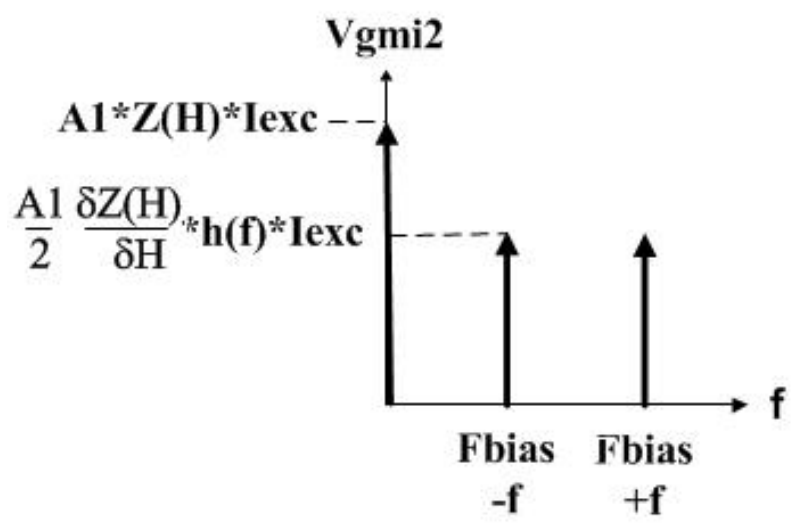

Figure 7. Frequency domain of GMI signal (Vgmi2) after multiplication by clock at $f_{\text {exc }}$.

Fig. 10), using the electronic principle described previously, exhibits a constant gain from DC up to $20 \mathrm{~Hz}$. The NEMI of the GMI part (green plot in Fig. 10d) is about 600 pT/ $\sqrt{\mathrm{Hz}}$ at $1 \mathrm{~Hz}$, far from the best fluxgate one, mentioned by Ripka (2003), while the NEMI for the inductive part of the hybrid sensor when the GMI is switched OFF (blue plot in Fig. 10d)

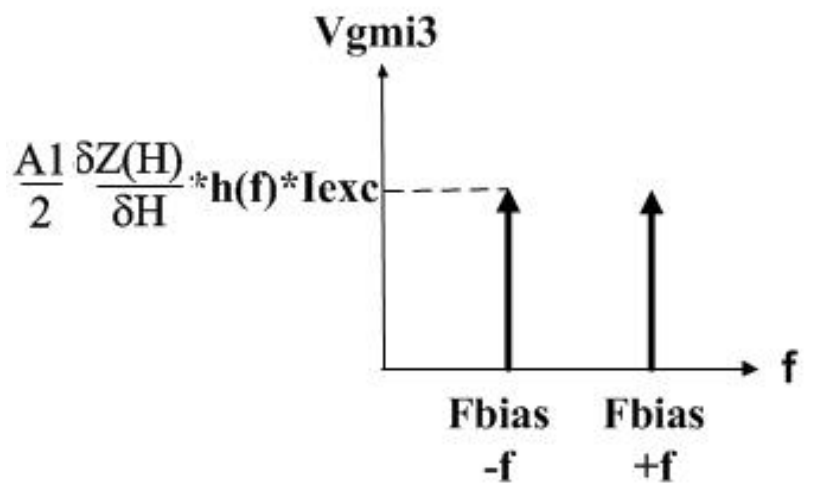

Figure 8. Frequency domain of GMI signal (Vgmi3) after highpass filter.

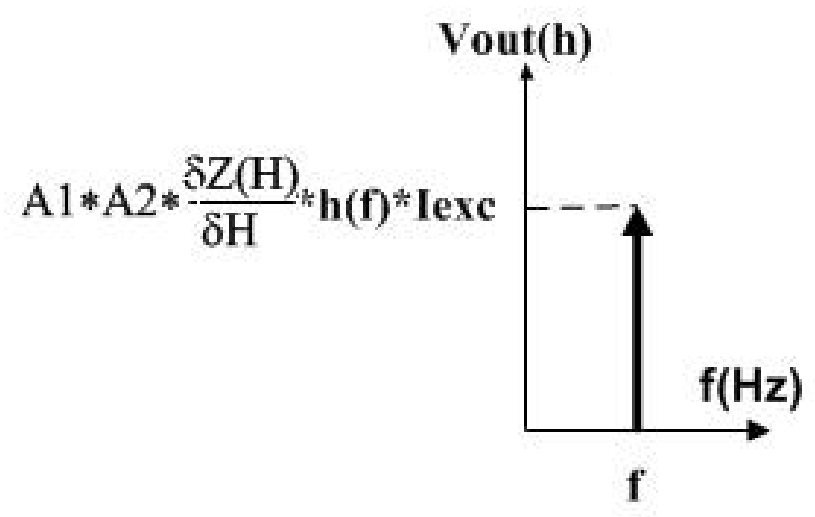

Figure 9. Frequency domain of GMI signal (Vgmi4) after multiplication by clock at $f_{\text {bias }}$, amplification (A2) and low-pass filtering.

reaches $100 \mathrm{fT} / \sqrt{\mathrm{Hz}}$ at $4 \mathrm{kHz}$. Concerning the GMI part, many possibilities of improvement have to be investigated, some of them could be inspired from Dumay et al. (2012); namely, turn number, increase of excitation frequency and 

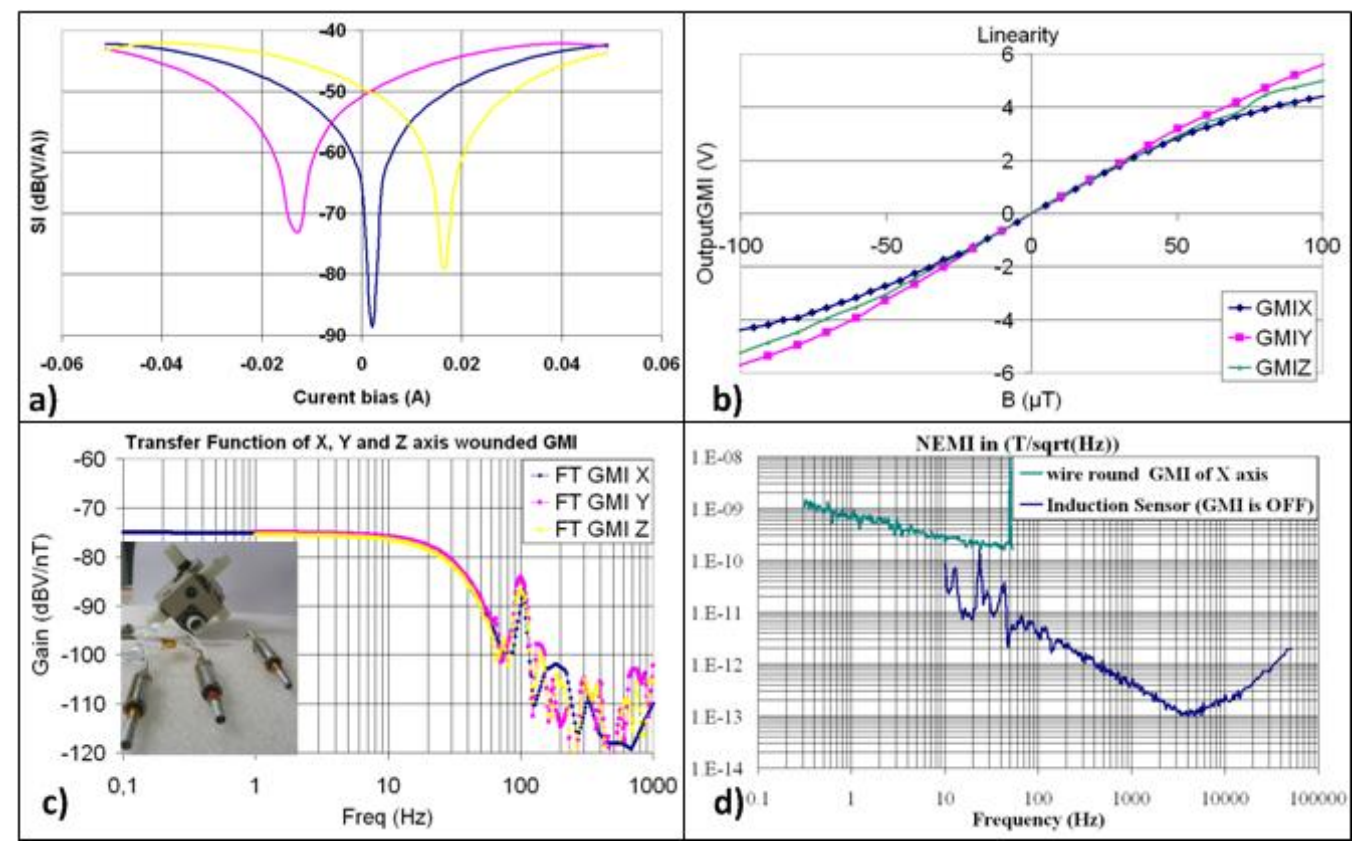

Figure 10. (a) Performances of the tri-axes hybrid magnetometer. The intrinsic sensitivity $S_{\mathrm{I}}$ has been measured when the sensor axis is perpendicular to an external DC magnetic field (black curve) and when it is either parallel or anti-parallel (yellow and magenta curves), more precisely $20 \log \left(S_{\mathrm{I}}\right)$ is plotted in frame (a), frame (b) demonstrates the linearity of the sensor with respect to the external DC magnetic field, frame (c) shows the transfer functions $20 \log ($ Gain), Gain being measured in $V / n T$, frame (d) is a plot of the noise equivalent magnetic induction in $\mathrm{T} / \sqrt{\mathrm{Hz}}$ between 0.3 and $50 \mathrm{~Hz}$ (in green) for the GMI part (the peak at $50 \mathrm{~Hz}$ being due to the network power supply) and between $10 \mathrm{~Hz}$ and $50 \mathrm{kHz}$ (in blue) for the induction sensor part.

input voltage noise reduction. The induction sensor part of the hybrid sensor becomes more sensitive than the GMI sensor from a few $\mathrm{Hz}$. One should notice an interesting property of the electronic principle which is the intrinsic linearization of the output voltage despite a strong variation of the intrinsic sensitivity around a bias field value. Thanks to the symmetry of $S_{\text {I }}$ curves (Fig. 10a) and the alternating biasing, the variation of $S_{\text {I }}$ due to the shift of the positive bias field when $H$ field is measured is compensated by an opposite variation of $S_{\text {I }}$ for the negative bias field. Concerning the induction sensor it should be noticed that the strong signature of the alternating bias of the wire-wound GMI sensor remains at the fundamental of bias magnetic field $\left(f_{\text {bias }}=100 \mathrm{~Hz}\right)$ but it does not affect too much the induction sensor transfer function above a few $100 \mathrm{~Hz}$ since the reduction of the $\mathrm{AC}$ disturbance from the wire-wound GMI is more efficient when frequency increases thanks to the aluminium tube which acts as a high-pass filter magnetic shielding. However the induction sensor NEMI was worst in the presence of the GMI because of residual disturbing fields (mainly lines at the bias field harmonics frequencies). The way to reduce it would be to increase bias frequency out of the induction sensor frequency range. The performance of the hybrid magnetometer is summarized in Table 1.
Table 1. Performance of the tri-axes hybrid magnetometer combining a search coil and a wire-wound GMI.

\begin{tabular}{ll}
\hline Frequency range & DC $-20 \mathrm{kHz}$ \\
\hline Sensitivity & $200 \mathrm{kV} \mathrm{T}^{-1}$ \\
Linearity range & $\pm 25 \mu \mathrm{T}$ \\
NEMI $(1 \mathrm{~Hz})$ & $600 \mathrm{pT} / \sqrt{\mathrm{Hz}}$ \\
NEMI $(4 \mathrm{kHz})$ & $100 \mathrm{fT} / \sqrt{\mathrm{Hz}}$ \\
Power consumption & $500 \mathrm{~mW}$ \\
Mass (preamplifier box) & $550 \mathrm{gr}$ \\
Mass (sensors) & $500 \mathrm{gr}$ \\
Size (tri-axis sensors) & $120 \mathrm{~mm} \times 120 \mathrm{~mm} \times 120 \mathrm{~mm}$ \\
\hline
\end{tabular}

\section{Instrument and measurement discussion}

The NASA sounding rocket CHARM-II was launched successfully from Poker Flat, Alaska, on 16 February 2010. Figure 11 shows the launch of the rocket, where the green light in the lower right is a wonderful aurora borealis. The prototype embedded in the scientific payload has operated nominally. The measured DC magnetic field from the wire-wound GMI has been used to reconstruct the magnetic field line along the rocket trajectory. The measured magnetic field line fits well with the computed Earth magnetic field (using the 


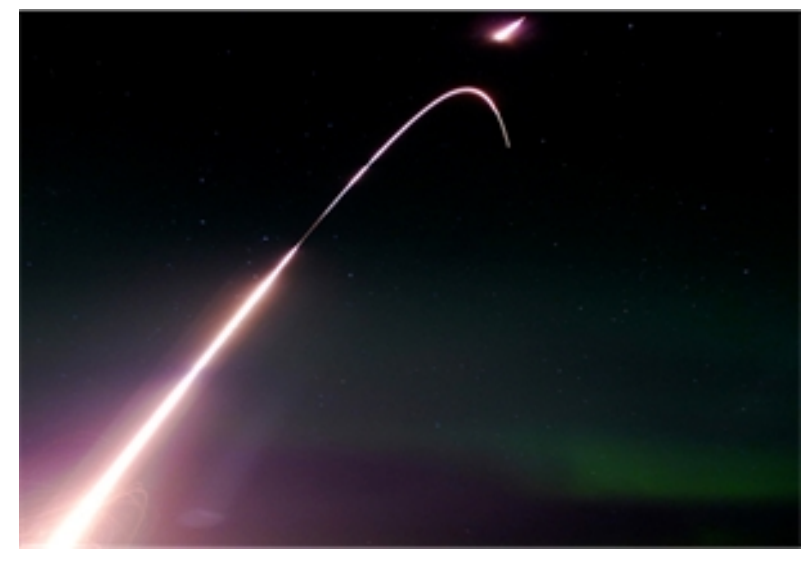

Figure 11. The launch of the CHARM-II rocket from Poker Flat (Alaska) during an aurora borealis (Copyright Micah P. Dombrowski).

International Geomagnetic Reference Field model) except at the time of the boom deployment where a jump of about $20 \%$ of the Earth's magnetic field magnitude is obtained. The origin of this jump has not been elucidated and for this reason the measurement of the Earth's magnetic field is not reported in the paper.

The combination of the inductive sensor with a wirewound racetrack GMI sensor allows one to cover a frequency range measurement from quasi-DC up to a few $\mathrm{kHZ}$. The major drawback of this solution of hybrid sensor is the residual flux leakage of both excitation and bias fields which disturbs the induction sensor part by adding lines at harmonics of the bias frequency. That problem could be overcome by investigating an increase of the bias frequency. Moreover, the flux of the magnetic field to be measured is divided into two parts: one part is diverted into the ferromagnetic ribbon of the GMI while the other part is diverted into the ferromagnetic core of the search coil. That reduces the efficiency if we compare to the GMI or induction sensor working separately. For these reasons, possibly combined to a magnetically quiet launch period, the instrument did not measure the magnetic component of electromagnetic waves during the flight. Finally, the hybridization of the induction sensor with a wire-wound GMI sensor is not well suited for the induction sensor and the NEMI at $1 \mathrm{~Hz}$ remains much higher than the one of a fluxgate. Nevertheless the principle of the wire-wound GMI sensor and its electronics, presented in this paper, are easy to implement and allow to build, in a pedagogic way, an efficient magnetometer.
Acknowledgements. This research work has been funded by the Research and Development Programme of CNES, the Space French Agency. The authors are grateful to J. Labelle for their participation in the NASA CHARM-II rocket experiment. The authors would also like to thank Rabah Ikhlef and Zaki Grig Ahcene, master students who have participated with great enthusiasm on the design, tests and manufacturing of the magnetic sensors.

Edited by: A. Schütze

Reviewed by: two anonymous referees

\section{References}

Aharoni, A.: Demagnetizing factors for rectangular ferromagnetic prisms, J. Appl. Phy., 83, 3432-3434, 1998.

Belevitch, V.: The lateral skin effect in a flat conductor, Philips tech Rev., 32, 221-231, 1971.

Coillot, C. and Leroy, P.: Induction Magnetometers: Principle, Modeling and Ways of Improvement, Magnetic Sensors - Principles and Applications, edited by: Kuang, K., ISBN: 978-953-510232-8, InTech, 2012.

Dufay, B., Saez, S., Dolabdjian, C., Yelon, A., and Ménard, D.: Characterizaion of an optimized off-diagonal GMI based magnetometer, IEEE Sensors, 99, 379-388, 2012.

Garcia-Arribas, A., Barandiaran, J. M., and de Cos, D.: Finite element method calculations of GMI in thin films and sandwiched structures: size and edge effects, Journal of Magnetism and Magnetic Materials, 320, e4-e7, 2008.

Harrison, H. P., Turney, G. P., Rowe, H., and Gollop, H.: The electrical properties of High permeability Wires Carrying Alternating Current, Proc. Royal. Soc., 157, 451-479, 1936.

Hika K. et al., Magneto-Impedance in Sandwich Film for Magnetic Sensor Heads, IEEE Trans. Magn, Vol. 32, pp. 4594-4596, 1996.

Leuzinger, A. and Taylor, A.: Magneto-Inductive Technology Overview, PNI white paper, February 2010.

Malatek, M. and Kraus, L.: Off-diagonal GMI sensor with stressannealed amorphous ribbon, Sensors and Actuators, 64, 41-45, 2010.

Moutoussamy, J.: Nouvelles solutions de capteurs effet de magneto-impdance gé ante: Principe, Modélisation et Performances, $\mathrm{PhD}$ dissertation, Ecole Normale Supérieure de Cachan (France). http://tel.archivesouvertes.fr/docs/00/50/57/44/ PDF/Moutoussamy2009.pdf, 2009.

Moutoussamy, J., Coillot, C., Alvès, F., and Chanteur, G.: Feasibility of a giant magneto-impedance sandwich magnetometer for space applications, IEEE Sensors Conference (Atlanta-USA), 1013-1016, October 2007.

Moutoussamy, J., Coillot, C., Alvès, F., and Chanteur, G.: Longitudinal and transverse coiled giant magnetoimpedance transducers: principle, modelling and performances, Transducer 09 (Colorado-USA), June 2009.

Morikawa, T., Nishibe, Y., and Yamadera, H.: Giant MagnetoImpedance Effect in Layered Thin Films, IEEE Transactions on Magnetics, 33, 4367-4372, 1997.

Panina, L. V. and Mohri, K.: Magneto-impedance effect in amorphous wires, Appl. Phys. Lett., 65 pp., 1189-1191, 1994a.

Panina, L. V., Mohri, K., Bushida, K., and Noda, M.: Giant magneto-impedance and magneto- inductive effects in amorphous alloys, J. Appl. Phys., 76, 6198-6203, 1994b. 
Paperno, R., Weiss, E., and Plotkin, A.: A Tube-Core Orthogonal Fluxgate Operated in Fundamental Mode, IEEE Transactions on Magnetics, 44, 4018-4021, 2008.

Ripka, P.: Advances in Fluxgate sensors, Sensors and Actuators A, 106, 8-14, 2003.

Sasada, I.: Symmetric response obtained with an orthogonal fluxgate operated in fundamental mode, IEEE Trans. Magn., 38, 3377-3379, 2002.

Samara, M., LaBelle, J., Kletzing, C. A., and Bounds, S. R.: Electrostatic upper hybrid waves where the upper hybrid frequency matches the electron cyclotron harmonic in the auroral ionosphere, Geophys. Res. Lett., 31, L22804, doi:10.1029/2004GL021043, 2004.
Seran, H. C. and Fergeau, P.: An optimized low frequency three axis search coil for space research, Rev. Sci. Instrum., 76, pp. 044502-1 044502-11, 2005.

Tumanski, S.: Induction coil sensors - A review, Meas Sci. Technol., 18, R31-R46, 2007.

VAC: Vacuumschmelze GMBH and Co Kg, Soft Magnetic Materials and Semi-Finished Products, Edition 2002. 